\title{
A Low fT3 Level as a Prognostic Marker in Patients with Acute Myocardial Infarctions
}

\author{
Baowei Zhang, Wenhui Peng, Chaofan Wang, Weiming Li and Yawei Xu
}

\begin{abstract}
Objective To investigate the association between low free triiodothyronine (fT3) levels and the severity and prognosis of patients with acute myocardial infarction.

Methods A total of 501 patients with acute myocardial infarctions were enrolled in our study. The circulating levels of thyroid hormones and clinical parameters were assayed. The patients were categorized into either the low fT3 group or the normal fT3 group according to the fT3 level on admission. All patients underwent a follow-up for $10 \pm 2$ months for mortality from any cause and the occurrence of any adverse major cardiac events (MACE).

Results There were 171 patients in the low fT3 group (fT3<3.5 pmol/L) and 330 patients in the normal fT3 group ( $\geq 3.5 \mathrm{pmol} / \mathrm{L}$ ). During the follow-up period, 33 patients died $(6.6 \%)$ and the overall survival rates were $86.0 \%$ and $97.3 \%$ in patients with a low fT3 level and a normal fT3 level, respectively. The rates of MACE were $66.7 \%$ and $45.5 \%$ in the patients with and those without low fT3 levels, respectively. Using a multivariable Cox proportional hazards model, the fT3 level was found to be the most important predictor of cumulative death and MACE (hazard ratio [HR] for death: 0.142, $\mathrm{p}<0.001$ and HR for major adverse cardiac events: $0.748, \mathrm{p}=0.007$ ). A Kaplan-Meier analysis revealed that those patients with low fT3 levels had higher rates of MACE and death.

Conclusion A low fT3 level, a common phenomenon in patients with acute myocardial infarctions, is a strong predictor of short-term and long-term poor prognoses in patients with acute myocardial infarctions.
\end{abstract}

Key words: acute myocardial infarction, low free triiodothyronine level, prognosis

(Intern Med 51: 3009-3015, 2012)

(DOI: 10.2169/internalmedicine.51.7902)

\section{Introduction}

There is a close relationship between thyroid hormones (THs) and the cardiovascular system (1). As the principal bioactive hormone, triiodothyronine (T3) exerts many effects on myocardial contractility, the resistance of arterioles in the peripheral circulation and cardiovascular hemodynamics through the modulation of the transcription of target genes in the cardiovascular system such as myosin heavy chain, phospholamban, sarcoplasmic reticulum $\mathrm{Ca}^{2+}$-ATPase, $\mathrm{Na}^{+}$/ $\mathrm{Ca}^{2+}$ exchanger and so on (2).

TH changes are always combined with changes in markers of cardiovascular hemodynamic instability, such as low fT3 levels, that are often observed in patients with cardio- vascular diseases such as coronary artery disease (CAD) and heart failure. Inflammation, hypoxia and hemodynamic instability are considered to be the most important mechanisms underlying a low fT3 state (3). In patients with acute myocardial infarctions (AMIs), inflammation and hypoxia are present in the heart and peripheral tissues, which are important mechanisms underlying the low fT3 levels. Therefore, there is a close relationship between low fT3 levels and AMIs. However, few studies have so far investigated the relationship between low fT3 levels and the prognosis of patients with AMIs. The purpose of our study was to investigate the association between low fT3 levels and the severity and prognosis of patients with AMIs. 


\section{Materials and Methods}

\section{Study population}

The study protocol was approved by the hospital Ethics Committee. A total of 561 consecutive patients were diagnosed with AMI in the Department of Cardiology, Shanghai Tenth People's Hospital between June 2008 and June 2010. The diagnosis of AMI was made according to the guidelines of the ACC/AHA for the management of AMI, which include: typical chest pain, ST segment elevation or new left bundle branch block (LBBB) and troponin $\mathrm{T}$ (TnT) level elevation (4). All of the patients underwent coronary angiography (CAG) to make a definitive diagnosis and to evaluate the severity of coronary artery disease (CAD). Patients excluded from this study were those with a history of thyroid diseases $(n=5)$, abnormal serum levels of thyrotropic stimulating hormone $(\mathrm{TSH})(\mathrm{n}=7)$, a history of therapy with amiodarone $(n=7)$, a history of heart failure $(n=13)$ and the presence of any predominant severe systemic diseases $(n=$ 28). The remaining 501 patients were included in this study.

\section{Biochemical investigation}

Blood samples were collected after overnight fasting during the first 24 hours after admission and stored at $-80^{\circ} \mathrm{C}$. The serum glucose and lipid profiles were measured (HITACHI 912 Analyser, Roche Diagnostics, Germany) as indicated previously (5). The levels of N-terminal Pro-Brain Natriuretic Peptide (NT-ProBNP) were measured with the Elecsys electro-chemiluminescent immunoassay (Roche Diagnostics Ltd. Rotkreuz, Switzerland).

We measured the serum levels of fT3, free tetraiodothyronine (fT4), thyroid stimulating hormone (TSH), total triiodothyronine (TT3) and total tetraiodothyronine (TT4) using chemiluminescence (Automatic Chemiluminescence Immune Assay System ACS 180 with related kits; Bayer, Berlin, Germany). The normal ranges for fT3, fT4, TSH, TT3 and TT4 are 3.5-6.5 pmol/L, 10.2-31 pmol/L, 0.35-5.5 mIU/ $\mathrm{L}, 1.2-3.4 \mathrm{nmol} / \mathrm{L}$ and $4-174 \mathrm{nmol} / \mathrm{L}$, respectively, in Shanghai, China (6).

\section{Coronary angiography}

CAG was performed using a standard Judkins technique or through a radial approach. Significant coronary artery disease was diagnosed visually if luminal diameter narrowing $\geq 50 \%$ was present in a major epicardial coronary artery. Left main coronary artery narrowing $\geq 50 \%$ was considered to indicate 2-vessel disease (7).

\section{Echocardiographic assessment}

All patients underwent transthoracic echocardiography by means of an echocardiograph equipped with a broadband transducer (Vivid $7^{\circledR}$, GE VingMed Ultrasound AS; Horten, Norway). Measurements of the left ventricle and left atrium were obtained from the parasternal long-axis and apical 4- chamber views, in accordance with standard criteria. The left ventricular ejection fraction (LVEF) was calculated using the modified Simpson rule in the apical 2- and 4chamber views.

\section{Follow-up}

Follow-up began the day of $\mathrm{TH}$ evaluation. Follow-up data were obtained in the following three ways: reviewing the patients' hospital records, interviewing the patients via telephone and examining the patients in outpatient clinics. The primary endpoint was cumulative death (death from any natural cause). The secondary endpoint was the incidence of major adverse cardiac events (MACE), defined as any of the following: cardiac death, rehospitalization for heart failure, nonfatal myocardial infarction or severe angina for coronary revascularization. The definition of cardiac death required the documentation of significant arrhythmia or cardiac arrest, death attributable to congestive heart failure or myocardial infarction in the absence of any other precipitating factors. Deaths caused by accidents were excluded (follow-up censored at the time of death). The mean follow-up duration was $10 \pm 2$ months.

\section{Statistical analysis}

Continuous variables are expressed as the mean \pm standard deviation. Categorical variables are presented as frequencies. In the univariate analysis, continuous variables were compared using Student's $t$-test (if homogeneity of variances was assumed) or the Mann-Whitney test (if homogeneity of variances was not met). Categorical data were analyzed using the $\chi^{2}$ or Fisher's exact test when appropriate. A linear regression analysis was performed to evaluate the relationship between the fT3 level and the NT-ProBNP level. Univariate and multivariate survival analyses were performed using the Cox proportional-hazards model to establish the combined risk of all causes of death and MACE for the variables assessed. Continuous variables (age, fT3, fT4, TT3, TT4, TSH, fasting serum glucose, TnT, NT-ProBNP, total cholesterol, high density lipoprotein, low density lipoprotein, serum creatinine, hemoglobin, white blood cells, C reactive protein, albumin, serum potassium, systolic/diastolic pressure, heart rate and LVEF) and dichotomized variables (sex and history of hypertension, diabetes or smoking) were entered into the model with their individual values or according to the presence (yes) or absence (no) of the variable, respectively. An automatic stepwise selection procedure using the maximum partial likelihood ratio $\chi^{2}$ statistic $\left(\chi^{2}\right.$ test) to enter $(\mathrm{p} \leq 0.05$ level) or remove $(\mathrm{p}>0.05$ level) a covariate into the model was used. The Kaplan-Meier method was used to analyze the timing of death or MACE during the follow-up. The statistical assessments were performed with the log-rank test, with values of $\mathrm{p}<0.05$ considered significant. The statistical analyses were performed using SPSS 13.0 (SPSS Inc., IL, USA) and a 2-sided probability level of $<0.05$ was taken to indicate significance. 
Table 1. Clinical Characteristics of Study Population

\begin{tabular}{|c|c|c|c|}
\hline \multirow[t]{2}{*}{ Characteristics } & Low fT3 & Normal fT3 & \multirow[t]{2}{*}{$\mathrm{p}$ value } \\
\hline & $(n=171,34.1 \%)$ & $(\mathrm{n}=330,65.9 \%)$ & \\
\hline Age (year) & $70 \pm 12$ & $68 \pm 12$ & 0.065 \\
\hline History of Hypertension, $\mathrm{n}(\%)$ & $96(56.1 \%)$ & $189(57.3 \%)$ & 0.808 \\
\hline Male sex, $n(\%)$ & $102(59.6 \%)$ & $243(73.6 \%)$ & 0.001 \\
\hline History of Diabetes, $n(\%)$ & $51(29.8 \%)$ & $102(30.9 \%)$ & 0.803 \\
\hline History of Smoking, $\mathrm{n}(\%)$ & $51(29.8 \%)$ & $162(42.5 \%)$ & $<0.001$ \\
\hline Fasting serum glucose $(\mathrm{mmol} / \mathrm{L})$ & $7.33 \pm 3.24$ & $6.81 \pm 2.36$ & 0.113 \\
\hline Total cholesterol $(\mathrm{mmol} / \mathrm{L})$ & $4.64 \pm 0.98$ & $4.54 \pm 1.13$ & 0.230 \\
\hline High density lipoprotein $(\mathrm{mmol} / \mathrm{L})$ & $1.11 \pm 0.24$ & $1.12 \pm 0.39$ & 0.489 \\
\hline Low density lipoprotein (mmol/L) & $2.43 \pm 0.98$ & $2.29 \pm 0.88$ & 0.097 \\
\hline Triglyceride $(\mathrm{mmol} / \mathrm{L})$ & $1.71 \pm 0.79$ & $1.79 \pm 1.13$ & 0.652 \\
\hline Serum creatinine (umol/L) & $90.23 \pm 29.99$ & $84.53 \pm 25.50$ & 0.181 \\
\hline Hemoglobin $(g / L)$ & $123.13 \pm 14.75$ & $126.73 \pm 16.88$ & 0.019 \\
\hline WBC $\left(10^{9} / \mathrm{L}\right)$ & $10.44 \pm 2.80$ & $9.67 \pm 2.84$ & 0.007 \\
\hline $\mathrm{C}$ reactive protein $(\mathrm{mg} / \mathrm{L})$ & $32.33 \pm 39.74$ & $25.66 \pm 34.31$ & 0.045 \\
\hline Totol protein $(\mathrm{g} / \mathrm{L})$ & $66.74 \pm 4.11$ & $67.03 \pm 4.51$ & 0.261 \\
\hline Albumin $(g / L)$ & $37.90 \pm 2.40$ & $38.01 \pm 3.01$ & 0.682 \\
\hline Serum potassium $(\mathrm{mmol} / \mathrm{L})$ & $3.80 \pm 0.61$ & $3.81 \pm 0.46$ & 0.334 \\
\hline Systolic pressure (mmHg) & $130.85 \pm 23.02$ & $132.44 \pm 20.34$ & 0.839 \\
\hline Diastolic pressure $(\mathrm{mmHg})$ & $76.65 \pm 12.87$ & $78.45 \pm 12.71$ & 0.183 \\
\hline Heart Rate (beats per min) & $78 \pm 12$ & $75 \pm 13$ & 0.039 \\
\hline Killip class on admission, $\mathrm{n}(\%)$ & & & 0.001 \\
\hline I & $91(53.2 \%)$ & $236(71.5 \%)$ & \\
\hline II & $58(33.9 \%)$ & $72(21.8 \%)$ & \\
\hline III & $20(11.7 \%)$ & $20(6.1 \%)$ & \\
\hline IV & $2(1.2 \%)$ & $2(0.6 \%)$ & \\
\hline Thrombolytic therapy, n(\%) & $28(16.4 \%)$ & $57(17.3 \%)$ & 0.799 \\
\hline Primary PCI, $\mathrm{n}(\%)$ & $126(73.7 \%)$ & $239(72.4 \%)$ & 0.764 \\
\hline fT3 $(\mathrm{pmol} / \mathrm{L})$ & $3.07 \pm 0.28$ & $4.15 \pm 0.47$ & $<0.001$ \\
\hline TT3 (nmol/L) & $1.38 \pm 0.32$ & $1.91 \pm 1.52$ & $<0.001$ \\
\hline fT4 $(\mathrm{pmol} / \mathrm{L})$ & $15.82 \pm 4.02$ & $16.14 \pm 2.73$ & 0.012 \\
\hline TT4 (nmol/L) & $93.08 \pm 20.57$ & $101.94 \pm 25.07$ & $<0.001$ \\
\hline TSH (mIU/L) & $1.25 \pm 0.96$ & $2.26 \pm 8.62$ & 0.328 \\
\hline
\end{tabular}

\section{Results}

\section{Basic characteristics}

Five hundred and one patients with an average age of $69 \pm$ 12 years were enrolled in our study. There were 171 patients with a low level of fT3 and 330 patients with a normal level of fT3 according to the reference values. One hundred and fifty four patients in the low fT3 group and 296 patients in the normal fT3 group received reperfusion therapy by means of primary PCI or thrombolytic therapy. One hundred and forty-three patients in the low fT3 group and 273 patients in the normal fT3 group underwent CAG during the acute phase of AMI and the remaining patients underwent CAG just before discharge. There were lower percentages of men and patients with a history of smoking in the low fT3 group; however, the percentage of patients in a high Killip class (Killip classes $>1$ ) was higher in the low fT3 group. The low fT3 group exhibited higher white blood cell counts $\left(10.44 \pm 2.80 \times 10^{9} / \mathrm{L}\right.$ vs. $\left.9.67 \pm 2.84 \times 10^{9} / \mathrm{L}, \mathrm{p}=0.007\right)$, levels of $\mathrm{C}$ reactive protein $(32.33 \pm 39.74 \mathrm{mg} / \mathrm{L}$ vs. $25.66 \pm 34.31 \mathrm{mg} /$ $\mathrm{L}, \mathrm{p}=0.045)$ and heart rates on admission $(78 \pm 12$ beats per minute vs. $75 \pm 13$ beats per minute, $\mathrm{p}=0.039)$ than the normal fT3 group. However, the levels of hemoglobin were lower in the low fT3 group $(123.13 \pm 14.75 \mathrm{~g} / \mathrm{L}$ vs. $126.73 \pm$ $16.88 \mathrm{~g} / \mathrm{L}, \mathrm{p}=0.019)$. There were no significant differences in age, serum creatinine, lipid levels, fasting serum glucose, total protein, albumin, blood pressure on admission or history of hypertension or diabetes between the two groups (Table 1).

\section{Differences in NT-ProBNP, TnT, LVEF and other pa- rameters}

We found a negative correlation between the level of fT3 and logarithm NT-ProBNP (Ig NT-ProBNP) in the whole cohort $(\mathrm{r}=-0.319, \mathrm{p}<0.001$, Fig. 1). As indices of myocardial damage and predictors of prognoses in patients with AMI (8), the levels of TnT and NT-ProBNP were higher in the low fT3 group than in the normal fT3 group $(5.33 \pm 5.77$ $\mathrm{ng} / \mathrm{mL}$ vs. $3.79 \pm 4.51 \mathrm{ng} / \mathrm{mL} ; 5,234 \pm 6,634 \mathrm{pg} / \mathrm{mL}$ vs. $2,850 \pm$ $4,089 \mathrm{pg} / \mathrm{mL}$, respectively, all $\mathrm{p}<0.001)$. The length of hospital stay, an index of disease severity, was longer in the low fT3 group $(14 \pm 6$ days vs. $11 \pm 6$ days, $\mathrm{p}<0.001)$. However, there were no significant differences between the two groups in the LVEF or other parameters (Table 2).

\section{Severity of coronary artery disease}

We assessed the severity of coronary artery disease according to the number of diseased vessels by means of 


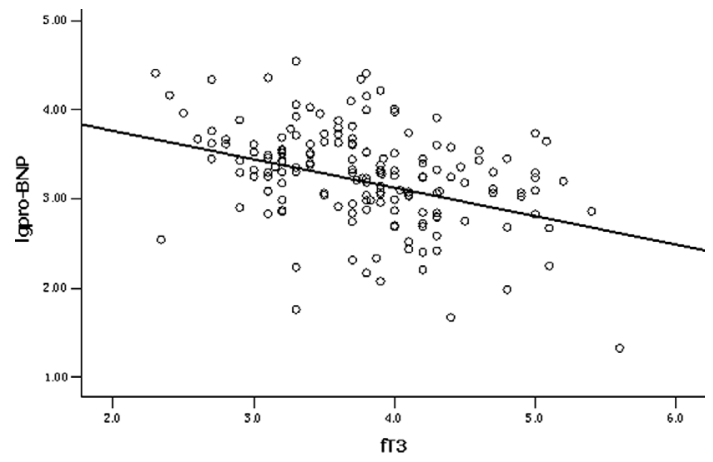

Figure 1. The linear correlation between the fT3 level and Ig NT-ProBNP. A linear regression analysis was performed to evaluate the relationship between the fT3 level and NT-ProBNP in the entire population. An inverse correlation was found between the fT3 level and the logarithm of NT-ProBNP, which indicates that a low fT3 level is a predictor of a poor prognosis in patients with AMIs.

Table 3. Severity of Coronary Disease

\begin{tabular}{lrrr}
\hline & Low fT3 group & Normal fT3 group & p value \\
\cline { 2 - 3 } & $(\mathrm{n}=171,34.1 \%)$ & $(\mathrm{n}=330,65.9 \%)$ & \\
\hline Diseased vessels & & & $<0.001$ \\
1-vessel, n (\%) & $27(15.8 \%)$ & $129(39.1 \%)$ & \\
2-vessel, n (\%) & $69(40.4 \%)$ & $118(35.8 \%)$ & \\
$\geq 3$-vessel, n (\%) & $75(43.9 \%)$ & $83(25.2 \%)$ & \multirow{2}{*}{0.058} \\
LMT lession, n (\%) & $9(5.3 \%)$ & $7(2.1 \%)$ & 0.895 \\
Culprit Vessel & & & \\
LAD, (n, \%) & $71(41.5 \%)$ & $139(42.1 \%)$ & \\
LCX, (n, \%) & $48(28.1 \%)$ & $97(29.4 \%)$ & \\
RCA, (n, \%) & $52(30.4 \%)$ & $94(28.5 \%)$ & \\
Baseline TIMI flow & & & \\
0, (n, \%) & $101(70.6 \%)$ & $165(60.2 \%)$ & \\
1, (n, \%) & $17(11.9 \%)$ & $32(11.7 \%)$ & \\
$2,(\mathrm{n}, \%)$ & $11(7.7 \%)$ & $47(17.2 \%)$ & \\
$3,(\mathrm{n}, \%)$ & $14(9.8 \%)$ & $30(10.9 \%)$ & \\
\hline LMT: Left Main Trunk, LAD: Left Anterior Descending, LCX: left Circumflex \\
Artery, RCA: Right Coronary Artery, TIMI: Thrombolysis in Myocardial Infarction
\end{tabular}

CAG. The low fT3 group had a higher percentage of patients with $\geq 3$ diseased vessels $(43.9 \%$ vs. $25.2 \%)$ and a lower percentage of patients with a single diseased vessel (15.8\% vs. $39.1 \%)$. However, there were no significant differences in the percentage of patients with two diseased vessels between the two groups. The low fT3 group showed a trend toward higher rates of left main trunk (LMT) lesions and lower baseline TIMI flow grades; however, the differences were not significant. In addition, the culprit vessels in the two groups were similar (Table 3).

\section{Correlation between fT3 and the primary endpoints}

Five hundred and one patients were successfully followed up for $10 \pm 2$ months. During the first 30 days, there were 13 deaths in the low fT3 group; however, no deaths occurred in the normal fT3 group. Twenty-seven patients in the low fT3 group and 26 patients in the normal fT3 group experienced MACE (15.6\% vs. $7.9 \%, p=0.008)$. During the long-term follow-up, $117(68.4 \%)$ patients in the low fT3 group and
Table 2. Differences of pro-BNP, TnT, LVEF and Other Parameters

\begin{tabular}{lrrr}
\hline Characteristics & Low fT3 & Normal fT3 & p value \\
\cline { 2 - 3 } & $(\mathrm{n}=171,34.1 \%)$ & $(\mathrm{n}=330,65.9 \%)$ & \\
\hline Troponin T $(\mathrm{ng} / \mathrm{mL})$ & $5.33 \pm 5.77$ & $3.79 \pm 4.51$ & $<0.001$ \\
Pro-BNP $(\mathrm{pg} / \mathrm{mL})$ & $5,234 \pm 6,634$ & $2,850 \pm 4,089$ & $<0.001$ \\
LVEF $(\%)$ & $55.42 \pm 12.29$ & $57.49 \pm 11.94$ & 0.086 \\
SD $(\mathrm{mm})$ & $35.40 \pm 6.88$ & $35.29 \pm 7.92$ & 0.402 \\
DD $(\mathrm{mm})$ & $49.81 \pm 8.00$ & $50.64 \pm 6.41$ & 0.605 \\
LAD $(\mathrm{mm})$ & $45.60 \pm 6.75$ & $44.52 \pm 5.33$ & 0.100 \\
Length of stay (days) & $14 \pm 6$ & $11 \pm 6$ & $<0.001$ \\
\hline
\end{tabular}

Pro-BNP: Pro B-type Natriuretic Peptide, LVEF: Left Ventricular Ejection Fraction, SD: Systoliv Diameter, DD: Diastolic Diameter, LAD: Left Atrium Diameter

Table 4. Comparison of Endpoints between Low fT3 Group and Normal fT3 Group

\begin{tabular}{lrrr}
\hline Endpoints & Low fT3 & Normal fT3 & p value \\
\cline { 2 - 4 } & $(\mathrm{n}=171,34.1 \%)$ & $(\mathrm{n}=330,65.9 \%)$ & \\
\hline 30-days Death, n (\%) & $13(7.5 \%)$ & $0(0)$ & $<0.001$ \\
30-days MACE, n (\%) & $27(15.6 \%)$ & $26(7.9 \%)$ & 0.008 \\
1-year Death, n (\%) & $24(14.0 \%)$ & $9(2.7 \%)$ & $<0.001$ \\
1-year MACE, n (\%) & $114(66.7 \%)$ & $150(45.5 \%)$ & $<0.001$ \\
1-year Rehospitalization, n (\%) & $117(68.4 \%)$ & $183(55.5 \%)$ & 0.005 \\
\hline MACE: Major Adverse Cardiac Events & & & \\
& & &
\end{tabular}

$183(55.5 \%)$ patients in the normal fT3 group were rehospitalized for all causes, including cardiac and non-cardiac diseases $(p=0.005)$. The mortality and incidence of MACE were higher in the low fT3 group than in the normal fT3 group (14.0\% vs. $2.7 \%$ and $66.7 \%$ vs. $45.5 \%$, respectively; all $\mathrm{p}<0.001$ ) (Table 4). According to a multivariate analysis, the fT3 level was the strongest independent predictor of cumulative death and MACE (hazard ratio [HR] for death: $0.142, \mathrm{p}<0.001$ and HR for MACE: 0.748, p=0.007) (Tables 5, 6). One year Kaplan-Meier survival curves for cumulative deaths and the incidence of MACE in patients with low versus normal fT3 levels are shown in Figs. 2, 3.

\section{Discussion}

A low fT3 level is a common phenomenon in patients with serious illnesses, including cardiovascular diseases. Accumulating evidence suggests that a low fT3 level is a strong predictor of a poor prognosis in patients with chronic cardiovascular diseases, including stable angina pectoris (9), chronic heart failure (10) and so on (11). In this study, we found a high rate $(34.1 \%)$ of low fT3 levels in the patients with AMIs, similar to that observed in other cardiovascular diseases (12). The low fT3 group had a higher percentage of patients with three diseased vessels and a lower percentage of patients with a single diseased vessel. The serum levels of TnT and NT-ProBNP, which reflect the degree of cardiac injury, were higher in the low fT3 group. The mean hospital stay was also longer in the low fT3 group. These findings indicate that a low fT3 level is correlated with the severity of AMI. In the whole cohort, we found an inverse correla- 
Table 5. Univariate and Multivariate Analysis of Predictors of 1-Year Mortality

\begin{tabular}{lrrr}
\hline Variables & Hazard Ratio & $95 \%$ CI & p value \\
\hline Univariate analysis & & & \\
fT3 & 0.225 & $0.120-0.423$ & $<0.001$ \\
Smoking & 7.757 & $2.367-25.422$ & 0.001 \\
TT3 & 0.192 & $0.079-0.463$ & $<0.001$ \\
TT4 & 0.977 & $0.967-0.987$ & $<0.001$ \\
TSH & 1.031 & $1.017-1.045$ & 0.007 \\
TnT & 1.102 & $1.053-1.154$ & $<0.001$ \\
Pro-BNP & 2.214 & $1.104-4.442$ & 0.025 \\
LDL & 0.554 & $0.361-0.849$ & 0.007 \\
Heart Rate & 0.960 & $0.934-0.986$ & 0.003 \\
Multivariate analysis & & & \\
fT3 & 0.142 & $0.063-0.322$ & $<0.001$ \\
smoking & 7.996 & $2.370-26.976$ & 0.001 \\
TSH & 1.031 & $1.013-1.049$ & 0.001 \\
TnT & 1.145 & $1.086-1.208$ & $<0.001$ \\
HDL & 0.154 & $0.028-0.855$ & 0.032 \\
Diastolic pressure & 1.035 & $1.000-1.070$ & 0.049 \\
Heart rate & 0.931 & $0.896-0.969$ & $<0.001$ \\
\hline Tn T: Troponin T, TSH: Thyroid Stimulating Hormone, HDL: High Density \\
Lipoprotein, CI: Confidence Interval, Pro-BNP: Pro B-type Natriuretic \\
Peptide, TT3: Total T3, TT4:Total T4 & & \\
& & &
\end{tabular}

tion between the level of fT3 and the logarithm of NTProBNP, a traditional predictor of poor prognoses in patients with AMIs, indicating that a low fT3 level would also be a predictor of a poor prognosis in AMI patients. We also found more endpoint events, including cumulative deaths and MACE, in the low fT3 group. Additionally, the KaplanMeier survival curves revealed that the prognoses were poorer in the low fT3 group than in the normal fT3 group. In a previous study, Friberg found a higher level of serum reverse T3 (rT3) to be a predictor of a poor prognosis in patients with AMIs; however, they did not find a predictive value for fT3 (13). The reasons for the differences in results between our study and Friberg's study are not clear; however, improvements in treatment, the study populations and the histories of diseases might contribute to these differences.

The mechanisms underlying the low fT3 levels observed in AMI patients are not fully understood. It is accepted that decreased conversion from $\mathrm{T} 4$ to $\mathrm{T} 3$ in peripheral tissues due to inflammatory factors, hypoxia and so on result in low fT3 levels. Inflammatory cytokines might also contribute to the incidence of low fT3 levels after AMI, as a negative correlation between the serum levels of fT3 and Interleukin-6 (IL-6) in AMI patients was found in a previous study (14). IL-6 reduces type 1 iodothyronine deiodinase (D1) and D2 activity by inducing oxidative stress in peripheral tissues. At the same time, IL- 6 can increase the transcription of D3 proteins by activating the JAK/STAT pathway. All of these changes decrease the conversion of $\mathrm{T} 4$ to $\mathrm{T} 3$ and increase the degradation of T3 (15). Hypoxia in peripheral tissues is another factor contributing to the low fT3 levels. Increased hypoxia-induced factor- 1 activates D3 in the heart and other tissues, which might contribute to lowering the fT3 level (16). In addition, other factors such as decreased ac-
Table 6. Univariate and Multivariate Analysis of Predictors of 1-Year Rate of MACE

\begin{tabular}{lrrr}
\hline Variables & Hazard Ratio & $95 \%$ CI & p value \\
\hline Univariate analysis & & & \\
fT3 & 0.644 & $0.528-0.784$ & $<0.001$ \\
TT4 & 0.994 & $0.989-0.998$ & 0.006 \\
TnT & 1.032 & $1.010-1.054$ & 0.005 \\
Pro-BNP & 1.045 & $1.025-1.065$ & $<0.001$ \\
Systolic pressure & 0.994 & $0.988-0.999$ & 0.031 \\
Multivariate Analysis & & & \\
fT3 & 0.748 & $0.606-0.923$ & 0.007 \\
TnT & 1.027 & $1.005-1.050$ & 0.018 \\
Pro-BNP & 1.038 & $1.016-1.060$ & 0.001 \\
TT4 & 0.995 & $0.990-1.000$ & 0.037 \\
\hline
\end{tabular}

fT3: free T3, Tn T: Troponin T, Pro-BNP: Pro B-type Natriuretic Peptide, TT4: Total T4, MACE: Major Adverse Cardiac Events, CI: Confidence Interval

tivities of $\mathrm{TH}$ binding proteins and the short $\mathrm{T} 3$ half-life might be associated with the low T3 level.

A low fT3 level increases the incidence of short-term and long-term adverse cardiac events through several pathways. Thyroid hormones exert direct effects on hemodynamics, including increasing cardiac contractility, decreasing vascular resistance and so on. A low fT3 state after AMI worsens hemodynamics and promotes adverse cardiac events. A low fT3 state after AMI changes the transcription of many cardiac structural and functional genes, for example, decreasing $\alpha$-myosin heavy chain $(\alpha-\mathrm{MHC})$ and sarcoplasmic reticulum calcium-activated ATPase (SERCA2) mRNA and increasing $\beta$-MHC and phospholamban (PLB) mRNA (17). These changes in the expression of cardiac genes are also characteristic of pathological cardiac remodeling after myocardial infarction (18) and lead to decreased contractility of the myocardium, inhibited $\mathrm{Ca}^{2+}$ transport, a worsened diastolic function, calcium overload, myocardial stunning and reperfusion injury. Animal experiments also show that thyroid hormone replacement treatment can reverse the changes in the shape and geometry of cardiac myocytes that occur after AMI (19). In addition, thyroid hormones are powerful regulators of vasculature in the adult myocardium; therefore, a low fT3 state would inhibit neovascularization in cardiac tissue after AMI, which would accelerate cardiac pathologic remodeling and heart failure (20). THs have been shown to activate the pro-survival PI3K/Akt signaling pathway and suppress the pro-death P38/MAPK signaling pathway in cardiac cells following ischemia (21). These changes in a low fT3 state would accelerate pathological cardiac remodeling and worsen the cardiac function, which would lead to shortterm and long-term adverse cardiac events.

\section{Study limitations}

This study found that determining the fT3 levels in patients with AMI is an important way to identify high-risk patients. However, there are some limitations that need to be acknowledged. First, the patients enrolled in this study came from a single center, which might limit the extrapolation of our results. This is particularly true when considering the homogeneity of the population with regard to race. Second, 


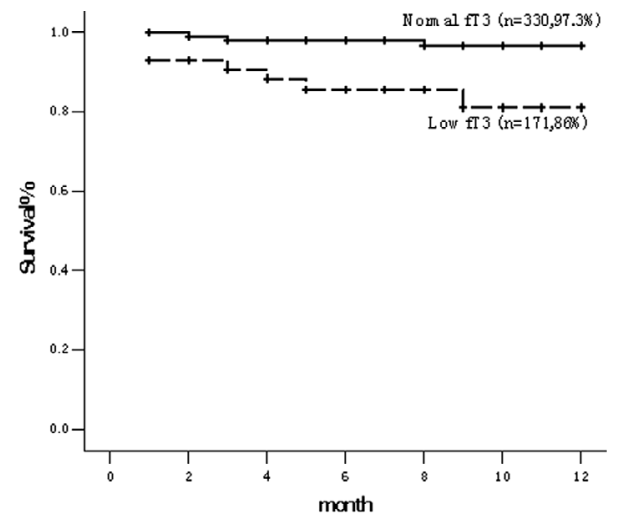

Figure 2. One year Kaplan-Meier survival curves for cumulative deaths in patients in the low fT3 level and normal fT3 level groups. The Kaplan-Meier method was used to analyze the timing of death during follow-up. The survival rate was higher in the normal fT3 group than in the low fT3 group, p $<0.001$.

this was a retrospective study and was consequently not immune to different types of bias. In addition, this study did not measure the fT3 level during follow-up. Therefore, we do not know whether the fT3 level changed after AMI. In addition, whether replacing thyroid hormones and raising fT3 levels into the normal range could help to improve the outcomes of the patients in the low fT3 group is unknown. These problems require further investigation with prospective studies.

\section{Conclusion}

The incidence of a low fT3 level is high in the population of patients with AMIs, and a low fT3 level is a strong predictor of a short- and long-term poor prognosis in AMI patients. Therefore, it might have great significance to investigate the level of TH on admission in patients with AMIs, which would help cardiologists to recognize high-risk patients.

The authors state that they have no Conflict of Interest (COI).

\section{Acknowledgement}

We thank all participants and nursing staff who cooperated with this study. This study was supported by grants from the National Natural Science Foundation of China (No. 30900520; 81070107) and the Shanghai Rising-Star Program (No. 10QA1405500).

\section{References}

1. Cini G, Carp A, Mechanick J, et al. Thyroid hormones and the cardiovascular system: pathophysiology and interventions. Biomed Pharmacother 63: 742-753, 2009.

2. Klein I, Ojamaa K. Thyroid hormone and the cardiovascular system. N Engl J Med 344: 501-509, 2001.

3. Pingitore A, Iervasi G, Barison A, et al. Early activation of an altered thyroid hormone profile in asymptomatic or mildly symptomatic idiopathic left ventricular dysfunction. J Card Fail 12: 520-

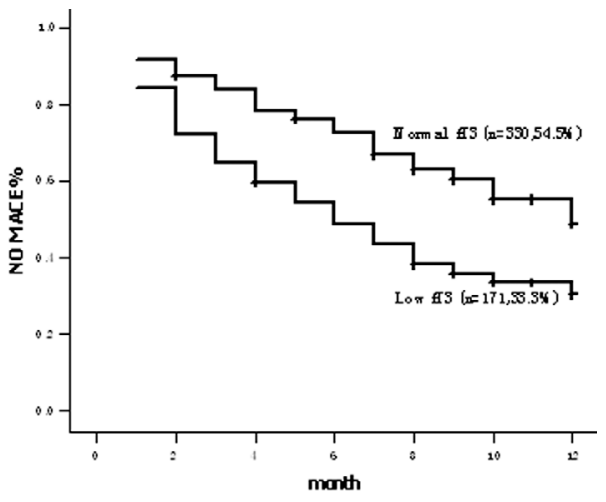

Figure 3. One year Kaplan-Meier survival curves for the incidence of MACE in patients in the low fT3 level and normal fT3 level groups. The Kaplan-Meier method was used to analyze the timing of the incidence of MACE during follow-up. The incidence of MACE was higher in the low fT3 group than in the normal fT3 group, $p<0.001$.

526, 2006.

4. Kushner FG, Hand M, Smith SC Jr, et al. 2009 focused updates: ACC/AHA guidelines for the management of patients with STelevation myocardial infarction (updating the 2004 guideline and 2007 focused update) and ACC/AHA/SCAI guidelines on percutaneous coronary intervention (updating the 2005 guideline and 2007 focused update) a report of the American College of Cardiology Foundation/American Heart Association Task Force on Practice Guidelines. J Am Coll Cardiol 54: 2205-2224, 2009.

5. Peng WH, Jian WX, Li HL, et al. Increased serum myeloidrelated protein $8 / 14$ level is associated with atherosclerosis in type 2 diabetic patients. Cardiovasc Diabetol 10: 41, 2011.

6. Fu W, Yang Y, Lin X, Luo K, Kang K. An approach to the correlation between vitiligo and autoimmune thyroiditis in Chinese children. Clin Exp Dermatol 35: 706-710, 2010.

7. Li HL, Peng WH, Cui ST, Hou L, Wei YD, Li WM. Vaspin plasma concentrations and mRNA expressions in patients with stable and unstable angina pectoris. Clin Chem Lab Med 49: 15471554, 2011.

8. Lorgis L, Zeller M, Dentan G, et al; RICO Survey Working Group. Prognostic value of N-terminal pro-brain natriuretic peptide in elderly people with acute myocardial infarction: prospective observational study. BMJ 338: b1605, 2009.

9. Coceani M, Iervasi G, Pingitore A, Carpeggiani C, L'Abbate A. Thyroid hormone and coronary artery disease: from clinical correlations to prognostic implications. Clin Cardiol 32: 380-385, 2009.

10. Pingitore A, Landi P, Taddei MC, Ripoli A, L'Abbate A, Iervasi G. Triiodothyronine levels for risk stratification of patients with chronic heart failure. Am J Med 118: 132-136, 2005.

11. Iervasi $G$, Landi $P$, Raciti $M$, et al. Low-T3 syndrome: a strong prognostic predictor of death in patients with heart disease. Circulation 107: 708-713, 2003.

12. Ascheim DD, Hryniewicz K. Thyroid hormone metabolism in patients with congestive heart failure: the low triiodothyronine state. Thyroid 12: 511-515, 2002.

13. Friberg L, Drvota V, Bjelak AH, Eggertsen G, Ahnve S. Association between increased levels of reverse triiodothyronine and mortality after acute myocardial infarction. Am J Med 111: 699-703, 2001.

14. Abo-Zenah HA, Shoeb SA, Sabry AA, Ismail HA. Relating circulating thyroid hormone concentrations to serum interleukins- 6 and10 in association with non-thyroidal illnesses including chronic renal insufficiency. BMC Endocrine Disorders 8: 1, 2008. 
15. Wajner SM, Goemann IM, Bueno AL, Larsen PR, Maia AL. IL-6 promotes nonthyroidal illness syndrome by blocking thyroxine activation while promoting thyroid hormone inactivation in human cells. J Clin Invest 121: 1834-1845, 2011.

16. Simonides WS, Mulcahey MA, Redout EM, et al. Hypoxiainducible factor induces local thyroid hormone inactivation during hypoxic-ischemic disease in rats. J Clin Invest 118: 975-983, 2008.

17. Ojamaa K, Kenessey A, Shenoy R, Klein I. Thyroid hormone metabolism and cardiac gene expression after acute myocardial infarction in the rat. Am J Physiol Endocrinol Metab 279: E1319E1324, 2000.

18. Dillmann W. Cardiac hypertrophy and thyroid hormone signaling.
Heart Fail Rev 15: 125-132, 2010.

19. Pantos $C$, Mourouzis I, Markakis K, et al. Thyroid hormone attenuates cardiac remodeling and improves hemodynamics early after acute myocardial infarction in rats. Eur J Cardiothorac Surg 32: 333-339, 2007.

20. Liu Y, Sherer BA, Redetzke RA, Gerdes AM. Regulation of arteriolar density in adult myocardium during low thyroid conditions. Vascul Pharmacol 52: 146-150, 2010.

21. Pantos C, Mourouzis I, Cokkinos DV. Thyroid hormone as a therapeutic option for treating ischaemic heart disease: from early reperfusion to late remodelling. Vascul Pharmacol 52: 157-165, 2010.

(C) 2012 The Japanese Society of Internal Medicine http://www.naika.or.jp/imonline/index.html 